\title{
Evaluation of hematological inflammatory markers in children and adolescents with attention deficit/hyperactivity disorder
}

\author{
Mehmet Akif Akınc1 ${ }^{1}$, Necati Uzun ${ }^{2}$ \\ Dr. Ali Kemal Belviranli Maternity and Children Hospital, Department of Child and Adolescent \\ Psychiatry, Konya, Turkey. akinci_mehmetakif@hotmail.com
}

\begin{abstract}
AIM: This study aims to investigate whether there is a relationship between attention-deficit/hyperactivity disorder (ADHD) and inflammation, using hematologic inflammatory parameters in a large sample of children and adolescents.

METHOD: This retrospective study comprises 347 children and adolescents with ADHD between 6 and 17 years of age who met the inclusion criteria as well as 205 healthy children and adolescents in the control group. Serum parameters such as white blood cell (WBC), neutrophil, lymphocyte, platelet, monocyte, eosinophil and basophil counts, mean platelet volume (MPV), and platelet distribution width (PDW) were recorded from complete blood count tests at the time of admission, while neutrophil lymphocyte ratio (NLR), platelet lymphocyte ratio (PLR), and other ratios were calculated based on these counts.

RESULTS: NLR, PLR, MPV, PDW, WBC and neutrophil counts were significantly higher in children and adolescents with ADHD than in the control group. In the ADHD group, NLR, WBC and neutrophil counts were significantly higher in girls than in boys.

CONCLUSIONS: The results of this study support the hypothesis that inflammation plays an important role in the complex pathophysiology of ADHD. In addition, our data confirm that easily obtainable and reliable hematological parameters can be used when investigating the role of inflammation in ADHD etiology (Tab. 4, Ref. 44). Text in PDF www.elis.sk

KEY WORDS: ADHD, inflammation, mean platelet volume, neutrophil/lymphocyte ratio, platelet/lymphocyte ratio.
\end{abstract}

\section{Introduction}

Attention-deficit/hyperactivity disorder (ADHD) is a neurodevelopmental disorder characterized by inattention, excessive mobility, and impulsiveness that do not confirm with the developmental level. It negatively affects community, school, or work-related activities (1). The worldwide prevalence of ADHD in children and adolescents is $5.29 \%$ (2). Based on manifestation, there are three subtypes of ADHD, namely inattentive predominant subtype, hyperactive/impulsive predominant subtype, and combined subtype. In a meta-analysis study, ADHD was found to be more commonly diagnosed in males, inattentive predominant subtype was more common in community-based studies, and combined subtype was predominantly observed in patients admitted to the clinic (3). ADHD has a complex etiology that involves an interaction between genetic and environmental factors (4). In

${ }^{1}$ Dr. Ali Kemal Belviranli Maternity and Children Hospital, Department of Child and Adolescent Psychiatry, Konya, Turkey, and ${ }^{2} \mathrm{Necmettin} \mathrm{Er-}$ bakan University Meram School of Medicine, Department of Child and Adolescent Psychiatry, Konya, Turkey

Address for correspondence: M.A. Akinci, MD, Dr. Ali Kemal Belviranli Maternity and Children Hospital Department of Child and Adolescent Psychiatry, Yeni Istanbul Cd. No: 32, 42285, Konya, Turkey.

Phone: +90 5066782680 addition, the exaggerated inflammatory response of the central nervous system to the damage that occurred during the prenatal period was assumed to be a potential cause of ADHD (5). Despite numerous studies carried out to date, the etiology of ADHD has not yet been fully clarified.

It is believed that the immune system has a role in the etiology of neurodevelopmental disorders. Studies show that pathophysiological mechanisms related to the immune system play a role in the emergence and progression of neurodevelopmental disorders (6). Various immune system-related disorders such as thyrotoxicosis, type 1 diabetes, autoimmune hepatitis, psoriasis, and ankylosing spondylitis have been associated with ADHD (7). The role of inflammation in has been proven also in neuropsychiatric disorders in line with increased interest in inflammation in recent years (8). In studies investigating the relationship between ADHD and inflammation, researchers showed that the levels of some cytokines (e.g., IFN-g) were elevated in ADHD patients and they found a correlation between elevated cytokines (proinflammatory and anti-inflammatory) and ADHD symptoms $(9,10)$. Additionally, the facts that cytokines may regulate basal ganglia and play a critical role in dopamine synthesis in the brain support the relationship between ADHD and cytokines (11).

Cytokines play an important role in both natural and cellular immune systems. However, the process of measuring peripheral cytokine levels is expensive because it employs complex proce- 
dures such as immunoassays. Based on this, recent studies on biomarkers have been focused on parameters obtained from the basic serum, such as blood cell count and blood cell ratios (12). A complete blood count is a readily available and inexpensive test that provides easily analyzable information about the body. Leukocytes play a critical role in inflammation since changes in leukocyte subgroups reflect the response of the immune system during the inflammatory state (13). The neutrophil/lymphocyte ratio (NLR) is a well-accepted clinical biomarker that can be easily obtained from blood and used for the evaluation of systemic inflammation (13). NLR was reported to be associated with the inflammatory response and prognosis in many clinical conditions such as autoimmune diseases and cardiovascular diseases $(14,15)$. It has also been reported that NLR value is significantly higher in psychiatric disorders such as schizophrenia, bipolar and depressive disorders in adults, as compared to the control group (16-18). In addition, an elevation in the neutrophil lymphocyte ratio has been shown to be present in child and adolescent psychiatric diseases $(19,20)$.

Platelets are cells that can interact with other circulating immune system cells and vascular endothelial cells. The activation of platelets and secreted proinflammatory cytokines (platelet-induced IL-1) play an important role in psychiatric disorders (21). The Platelet/lymphocyte ratio (PLR) is another biomarker derived from whole-blood count parameters, and considered to be an indicator of chronic low-grade inflammation (22). The mean platelet volume (MPV) is routinely given as part of the complete blood count (CBC) test. It is a machine-calculated parameter of the mean size of circulating platelets. In many studies, MPV has been shown to be a clinical marker of chronic low-grade inflammation $(23,24)$. Platelet distribution width (PDW) is another parameter that is available in nearly all laboratories. Changes in platelet functions and size have been reported to be associated with systemic inflammation, thus suggesting that PDW may be a valuable marker in predicting some disease outcomes $(25,26)$.

The relationship between hematologic inflammatory markers and ADHD has been studied in a small number of studies in children and adolescents. However, these studies had conflicting results. Avcil reported that NLR, PLR, MLR and MPV values were significantly higher in 82 children with ADHD, compared to the control group (27), whereas Binici et al. reported that NLR, PLR and MPV in 65 children with ADHD was not statistically different as compared to the control group (28). As far as we know, no previous studies have investigated eosinophil/lymphocyte ratio (ELR), basophil/lymphocyte ratio (BLR) and PDW, which are considered inflammatory markers in children and adolescents with ADHD. Thus, this study aims to clarify the relationship between ADHD and inflammation by using hematologic inflammatory markers in children and adolescents with ADHD.

\section{Materials and methods}

\section{Sample}

This retrospective study consisted of 347 children and adolescents with ADHD between 6 and 17 years of age that had been admitted to the Outpatient Child and Adolescent Psychiatry Cli- nic between February 2019 and April 2020. All data were taken from the hospital records of each patient stored in our hospital's electronic medical records database. These records include age, gender, BMI, diagnosis at the time of admission based on DSM-5 criteria, comorbid psychiatric disorders, other medical conditions and diseases, and laboratory test results. Both authors, who are child and adolescent psychiatrists, have examined the patients' files, and consensus was reached in all cases. This study was approved by the ethics committee of Karatay University (Date: 22 May 2020, number: 2020/019) and Konya Provincial Directorate of Health (Date: 29 July 2020, number: 86737044-806.01.03) Also, all procedures were conducted in accordance with the Declaration of Helsinki and local laws and regulations.

The inclusion criteria for the ADHD group were as follows: patients aged 17 and below (1); patients who met the DSM-5 diagnostic criteria for ADHD (2); no other psychiatric disorder (3); no psychiatric medication history at any period of life (4). The control group consisted of 205 healthy children and adolescents. The patient and control groups were similar in terms of age, gender and BMI. Exclusion criteria for both groups were as follows: concurrent psychiatric diagnoses (1); presence of an autoimmune disease, acute infection, systemic disease, epilepsy, allergic diseases, as well as other psychiatric, hematologic, endocrine, immunological, renal diseases, neurological and cardiological diseases (2); current use of all types of drugs (3); obesity, body mass index (BMI) more than $30 \mathrm{~kg} / \mathrm{m} 2$ (4); significant abnormalities in laboratory test results indicative of anemia, polycythemia, leukocytosis, leukopenia, lymphopenia, lymphocytosis, neutropenia, neutrophilia, thrombocytopenia or thrombocytosis (5).

\section{Procedure}

After collecting venous blood from patients into containers containing anticoagulant ethylene diaminetraacetic acid (EDTA), the Mindray BC-6800 auto hematology analyzer (BC-6800, Mindray, Shenzhen, China) was used to analyze hemograms. WBC, neutrophil, lymphocyte, platelet, monocyte, eosinophil, and basophil counts were recorded from complete blood counts at the time of admission, and NLR, PLR, MLR, ELR and BLR were calculated based on these measurements. Based on the data from hospital records and DSM-5 criteria, the patients were divided into three groups of subtypes, namely combined, inattentive and hyperactive/impulsive.

\section{Statistical analysis}

The data were analyzed using SPSS 20 software (IBM Corporation, Armonk, NY, USA). The data were presented as mean (standard deviation [SD]) for numerical variables and percentages for categorical variables. The Shapiro-Wilk normality test was used to determine whether the variables have a normal distribution. Chi-square $(\chi 2)$ test was used to compare categorical variables and frequencies. Continuous variables were expressed as means (SD). Student's t-test and one-way ANOVA and post-hoc Bonferonni test was used to compare the variables with normal distribution between two and three groups, respectively. However, the Mann-Whitney U test and the Kruskal-Wallis test was used to 
analyze non-normally distributed variables. All statistical analyses were performed within $95 \%$ confidence interval, and a $p$ value of $<0.05$ was considered statistically significant.

\section{Results}

This study included 347 children and adolescents with ADHD diagnosis (264 boys, 83 girls) with the mean age of $9.68 \pm 2.63$ years. The mean age of the 205 children and adolescents (150 boys, 55 girls) in the control group was $9.69 \pm 2.56$ years. There were no significant differences between the groups in terms of age, gender and BMI distribution $(p>0.05)$. Demographic data of the participants are shown in Table 1.

NLR and PLR values were significantly higher in children and adolescents with ADHD than in the control group $(\mathrm{p}<0.05)$. In addition, there was a significant difference between the two groups in terms of WBC, neutrophil count, PDW, and MPV $(\mathrm{p}<0.05)$. There was no significant difference between the groups in regard to the red blood cell distribution width (RDW), lymphocyte, monocyte, eosinophil, basophil, and platelet counts and MLR, ELR, and BLR values $(p>0.05)$. The comparison of serum parameters between groups is shown in Table 2 .

As presented in Table 3, 219 (63.1\%) patients were diagnosed with ADHD combined subtype, 93 (26.8\%) with ADHD inattentive subtype, and 35 (10\%) with ADHD hyperactive/impulsive subtype. Only MPV was statistically significant within hematological parameters among the ADHD subtypes (post hoc analysis, I $>\mathrm{H}$ ).

In the study group, as shown in Table 4, there were significant differences between NLR, hemoglobin $(\mathrm{Hb}), \mathrm{WBCs}$, and neutrophil counts between girls and boys, while other parameters were similar. of psychiatric diseases, hematological values that are considered new clinical markers of inflammation have been investigated in a variety of diseases. These values can easily be obtained from complete blood count tests and their analysis is cheap, repeatable and easily accessible. Inconsistent results were obtained in a limited number of studies evaluating these markers in ADHD, including NLR, PLR and MPV. Avcil's study reported an increase in these values, and suggested that they could be used as potential inflammatory markers in children with ADHD (27). Another study showed that the values of NLR, PLR and MPV in children with ADHD and healthy controls were similar (28). However, in the latter study, the children with additional psychiatric disorders were included in the ADHD group. The results of our study are consistent with the increased NLR, PLR and MPV values in children with ADHD. On the other hand, the increased values of MLR in the ADHD group shown in that study were not shown in our study. This may be attributable to the fact that the value of MLR loses its significance with an increase in the number of patients. In addition, in a study investigating the relationship between immune system cells and suicide attempts, no significant differences in MLR between the groups were found, which is supportive of our study

Tab. 1. Demographic and clinical characteristics of the participants.

\begin{tabular}{|c|c|c|c|c|c|}
\hline \multirow[t]{2}{*}{ Variables } & \multicolumn{2}{|c|}{$\begin{array}{l}\text { ADHD Group } \\
(\mathrm{n}: 347)\end{array}$} & \multicolumn{2}{|c|}{$\begin{array}{l}\text { Control Group } \\
(\mathrm{n}: 205)\end{array}$} & $\begin{array}{c}\text { Statistical } \\
\text { analysis }\end{array}$ \\
\hline & \multicolumn{2}{|c|}{$\mathrm{n}(\%)$} & \multicolumn{2}{|c|}{$\mathrm{n}(\%)$} & $\mathrm{p}$ \\
\hline \multirow[t]{2}{*}{ Boy/Girl } & \multicolumn{2}{|c|}{$264(76.1 \%) / 83(23.9 \%)$} & \multicolumn{2}{|c|}{$150(73.2 \%) / 55(26.8 \%)$} & 0.446 \\
\hline & Mean & $\mathrm{SD}$ & Mean & SD & $\mathrm{p}$ \\
\hline Age & 9.68 & 2.63 & 9.69 & 2.56 & 0.946 \\
\hline BMI & 18.67 & 2.51 & 18.71 & 2.40 & 0.847 \\
\hline
\end{tabular}

SD: standard deviation, BMI: body mass index $\left(\mathrm{kg} / \mathrm{m}^{2}\right)$

\section{Discussion}

In this study, which discusses the role of inflammation in the pathophysiology of ADHD, the inflammation status in pediatric and adolescent ADHD patients without medication and without comorbidity was assessed using parameters that can be detected by a complete blood count test. The results of the study showed that NLR, PLR, PDW and MPV values in the ADHD group were significantly higher compared to the control group. Also, WBC and neutrophil counts were higher in children and adolescents with ADHD than in healthy controls. As far as we know, this is the study with a relatively large sample size evaluating hematologic inflammation markers in children and adolescents with ADHD. It is also the first study that investigated particular markers of inflammation, such as ELR, BLR, and PDW, in children and adolescents with ADHD.

Along with the growing interest in the role of inflammation in the pathophysiology
Tab. 2. Comparison of hemogram parameters of the study groups.

\begin{tabular}{|c|c|c|c|c|c|}
\hline \multirow[t]{2}{*}{ Variables } & \multicolumn{2}{|c|}{$\begin{array}{c}\text { ADHD Group } \\
(\mathrm{n}: 347)\end{array}$} & \multicolumn{2}{|c|}{$\begin{array}{c}\text { Control Group } \\
(\mathrm{n}: 205)\end{array}$} & \multirow{2}{*}{$\begin{array}{c}\text { Statistical } \\
\text { Analysis } \\
\mathrm{p}\end{array}$} \\
\hline & Mean & $\mathrm{SD}$ & Mean & SD & \\
\hline$\overline{\mathrm{HGB}}$ & 13.48 & 0.93 & 13.47 & 1.02 & 0.963 \\
\hline HTC & 39.97 & 2.56 & 40.01 & 2.80 & 0.855 \\
\hline $\mathrm{RBC}$ & 4.96 & 0.34 & 4.92 & 0.35 & 0.182 \\
\hline RDW-CV & 13.44 & 0.74 & 13.45 & 0,82 & 0.826 \\
\hline WBC & 7.56 & 1.59 & 7.25 & 1.53 & 0.025 \\
\hline NEU & 4.06 & 1.25 & 3.69 & 1.11 & $<0.001$ \\
\hline LYM & 2.78 & 0.78 & 2.88 & 0.86 & 0.167 \\
\hline MON & 0.49 & 0.14 & 0.48 & 0.13 & 0.857 \\
\hline EOS & 0.19 & 0.13 & 0.17 & 0.12 & 0.140 \\
\hline BAS & 0.030 & 0.014 & 0.029 & 0.014 & 0.612 \\
\hline PLT & 297.54 & 60.97 & 290.66 & 75.80 & 0.270 \\
\hline PDW & 15.76 & 0.43 & 15.64 & 0.55 & 0.008 \\
\hline MPV & 8.72 & 0.88 & 8.50 & 0.85 & 0.004 \\
\hline NLR & 1.59 & 0.84 & 1.42 & 0.74 & 0.002 \\
\hline PLR & 114.26 & 37.01 & 110.12 & 44.66 & 0.014 \\
\hline MLR & 0.19 & 0.08 & 0.18 & 0.09 & 0.663 \\
\hline ELR & 0.071 & 0.054 & 0.063 & 0.050 & 0.077 \\
\hline BLR & 0.011 & 0.006 & 0.010 & 0.006 & 0.321 \\
\hline
\end{tabular}

SD: standard deviation, BMI: body mass index $\left(\mathrm{kg} / \mathrm{m}^{2}\right)$, HGB: hemoglobin $(\mathrm{g} / \mathrm{dL})$, HTC: hematocrit $(\%)$, RBC: red blood cell $(10 \% \mathrm{uL})$, RDW-CV: red cell distribution width $(\%)$, WBC: white blood cell $\left(10^{3} / \mathrm{uL}\right)$, NEU: neutrophile $\left(10^{3} / \mathrm{uL}\right)$, LYM: lymphocyte $\left(10^{3} / \mathrm{uL}\right)$, MON: monocyte $\left(10^{3} / \mathrm{uL}\right)$, EOS: eosinophil $\left(10^{3} / \mathrm{uL}\right)$, BAS: basophil $\left(10^{3} / \mathrm{uL}\right)$, PLT: platelet $\left(10^{3} / \mathrm{uL}\right)$, PDW: platelet distribution width $(\%)$, MPV: mean platelet volume, NLR: neutrophile/lymphocyte ratio, PLR: platelet/lymphocyte ratio, MLR: monocyte/lymphocyte ratio, ELR: eosinophil/lymphocyte ratio, BLR: basophil/lymphocyte ratio 
findings (29). One of the strengths of our study is the large sample size. The other strength lies in the fact that the present research is the first study that investigates ELR, BLR and PDW in children and

Tab. 3. Comparison of hemogram parameters among ADHD Subgroups.

\begin{tabular}{|c|c|c|c|c|c|c|c|c|c|}
\hline \multirow[t]{2}{*}{ Variables } & \multicolumn{2}{|c|}{$\begin{array}{l}\text { ADHD-C } \\
(\mathrm{n}: 219)\end{array}$} & \multicolumn{2}{|c|}{$\begin{array}{c}\text { ADHD-I } \\
\text { (n:93) }\end{array}$} & \multicolumn{2}{|c|}{$\begin{array}{c}\text { ADHD-H } \\
\text { (n:35) }\end{array}$} & \multicolumn{3}{|c|}{ Statistical Analysis } \\
\hline & Mean & $\mathrm{SD}$ & Mean & $\mathrm{SD}$ & Mean & $\mathrm{SD}$ & $\mathrm{F}$ & $\mathrm{p}$ & Post-Hoc \\
\hline Age & 9.68 & 2.60 & 9.90 & 2.72 & 9.06 & 2.52 & 1.315 & 0.270 & \\
\hline BMI & 18.66 & 2.48 & 18.65 & 2.47 & 18.79 & 2.58 & 2.028 & 0.157 & \\
\hline HGB & 13.49 & 0.98 & 13.52 & 0.88 & 13.30 & 0.64 & 0.728 & 0.483 & \\
\hline HTC & 39.94 & 2.64 & 40.33 & 2.56 & 39.16 & 1.79 & 2.687 & 0.070 & \\
\hline $\mathrm{RBC}$ & 4.94 & 0.32 & 5.01 & 0.39 & 5.00 & 0.33 & 1.257 & 0.286 & \\
\hline RDW-CV & 13.42 & 0.73 & 13.49 & 0.79 & 13.41 & 0,71 & 0.345 & 0.708 & \\
\hline WBC & 7.53 & 1.59 & 7.56 & 1.61 & 7.77 & 1.51 & 0.335 & 0.715 & \\
\hline NEU & 4.03 & 1.24 & 4.08 & 1.32 & 4.17 & 1.19 & 0.213 & 0.808 & \\
\hline LYM & 2.76 & 0.79 & 2.77 & 0.75 & 2.86 & 0.83 & 0.201 & 0.818 & \\
\hline MON & 0.49 & 0.14 & 0.49 & 0.13 & 0.45 & 0.16 & 1.572 & 0.209 & \\
\hline EOS & 0.20 & 0.14 & 0.16 & 0.09 & 0.19 & 0.116 & 2.919 & 0.166 & \\
\hline BAS & 0.030 & 0.016 & 0.030 & 0.013 & 0.028 & 0.011 & 0.575 & 0.563 & \\
\hline PLT & 296.27 & 64.87 & 297.81 & 55.32 & 304.74 & 50.07 & 0.291 & 0.748 & \\
\hline PDW & 15.75 & 0.44 & 15.75 & 0.31 & 15.83 & 0.64 & 0.450 & 0.638 & \\
\hline MPV & 8.71 & 0.91 & 8.88 & 0.79 & 8.38 & 0.90 & 4.158 & 0.016 & $\mathrm{I}>\mathrm{H}$ \\
\hline NLR & 1.59 & 0.84 & 1.56 & 0.64 & 1.70 & 1.26 & 0.346 & 0.708 & \\
\hline PLR & 113.93 & 34.80 & 112.72 & 29.18 & 120.45 & 61.82 & 0.577 & 0.562 & \\
\hline MLR & 0.19 & 0.09 & 0.18 & 0.07 & $0.16 \pm$ & 0.06 & 1.643 & 0.195 & \\
\hline ELR & 0.075 & 0.057 & 0.060 & 0.040 & 0.074 & 0.062 & 2.538 & 0.081 & \\
\hline BLR & 0.011 & 0.006 & 0.011 & 0.005 & 0.010 & 0.004 & 0.731 & 0.482 & \\
\hline
\end{tabular}

SD: standard deviation, BMI: body mass index $\left(\mathrm{kg} / \mathrm{m}^{2}\right)$, HGB: hemoglobin $(\mathrm{g} / \mathrm{dL})$, HTC: hematocrit $(\%)$, RBC: red blood cell $\left(10^{6} / \mathrm{uL}\right)$, RDW-CV: red cell distribution width (\%), WBC: white blood cell $\left(10^{3} / \mathrm{uL}\right)$, NEU: neutrophile $\left(10^{3} / \mathrm{uL}\right)$, LYM: lymphocyte $\left(10^{3} / \mathrm{uL}\right)$, MON: monocyte $\left(10^{3} / \mathrm{uL}\right)$, EOS: eosinophil $\left(10^{3} / \mathrm{uL}\right)$, BAS: basophil $\left(10^{3} / \mathrm{uL}\right)$, PLT: platelet $\left(10^{3} / \mathrm{uL}\right)$, PDW: platelet distribution width $(\%)$, MPV: mean platelet volume, NLR: neutrophile/lymphocyte ratio, PLR: platelet/lymphocyte ratio, MLR: monocyte/lymphocyte ratio, ELR: eosinophil/lymphocyte ratio, BLR: basophil/lymphocyte ratio

Tab. 4. Comparison of hemogram parameters among boys and girls with ADHD group.

\begin{tabular}{|c|c|c|c|c|c|}
\hline \multirow[t]{2}{*}{ Variables } & \multicolumn{2}{|c|}{$\begin{array}{c}\text { Boys } \\
(\mathrm{n}: 264)\end{array}$} & \multicolumn{2}{|c|}{$\begin{array}{c}\text { Girls } \\
(\mathrm{n}: 83)\end{array}$} & \multirow{2}{*}{$\begin{array}{c}\text { Statistical } \\
\text { Analysis }\end{array}$} \\
\hline & Mean & SD & Mean & SD & \\
\hline$\overline{\text { Age }}$ & 9.69 & 2.58 & 9.65 & 2.79 & 0.916 \\
\hline BMI & 18.70 & 2.43 & 18.57 & 2.76 & 0.681 \\
\hline HGB & 13.53 & 0.97 & 13.32 & 0.75 & 0.046 \\
\hline HTC & 39.98 & 2.67 & 39.94 & 2.20 & 0.900 \\
\hline $\mathrm{RBC}$ & 4.98 & 0.33 & 4.93 & 0.38 & 0.280 \\
\hline RDW-CV & 13.43 & 0.70 & 13.48 & 0,87 & 0.598 \\
\hline WBC & 7.43 & 1.56 & 7.99 & 1.61 & 0.005 \\
\hline NEU & 3.93 & 1.21 & 4.45 & 1.31 & 0.001 \\
\hline LYM & 2.77 & 0.75 & 2.81 & 0.86 & 0.642 \\
\hline MON & 0.48 & 0.14 & 0.50 & 0.13 & 0.207 \\
\hline EOS & 0.19 & 0.14 & 0.16 & 0.10 & 0.178 \\
\hline BAS & 0.031 & 0.015 & 0.028 & 0.013 & 0.108 \\
\hline PLT & 297.19 & 60.24 & 298.64 & 63.60 & 0.851 \\
\hline PDW & 15.76 & 0.45 & 15.75 & 0.39 & 0.779 \\
\hline MPV & 8.71 & 0.87 & 8.73 & 0.93 & 0.849 \\
\hline NLR & 1.55 & 0.80 & 1.74 & 0.94 & 0.015 \\
\hline PLR & 114.73 & 38.54 & 112.78 & 31.80 & 0.938 \\
\hline MLR & 0.18 & 0.07 & 0.19 & 0.10 & 0.272 \\
\hline ELR & 0.074 & 0.058 & 0.061 & 0.039 & 0.109 \\
\hline BLR & 0.011 & 0.006 & 0.010 & 0.005 & 0.100 \\
\hline
\end{tabular}

SD: standard deviation, BMI: body mass index $\left(\mathrm{kg} / \mathrm{m}^{2}\right)$, HGB: hemoglobin $(\mathrm{g} / \mathrm{dL})$, HTC: hematocrit $(\%)$, RBC: red blood cell $(10 \% \mathrm{uL}), \mathrm{RDW}-\mathrm{CV}$ : red cell distribution width $(\%)$, WBC: white blood cell $\left(10^{3} / \mathrm{uL}\right)$, NEU: neutrophile $\left(10^{3} / \mathrm{uL}\right)$, LYM: lymphocyte $\left(10^{3} / \mathrm{uL}\right)$, MON: monocyte $\left(10^{3} / \mathrm{uL}\right)$, EOS: eosinophil $\left(10^{3} / \mathrm{uL}\right)$, BAS: basophil $\left(10^{3} / \mathrm{uL}\right)$, PLT: platelet $\left(10^{3} / \mathrm{uL}\right)$, PDW: platelet distribution width $(\%)$, MPV: mean platelet volume, NLR: neutrophile/lymphocyte ratio, PLR: platelet/lymphocyte ratio, MLR: monocyte/lymphocyte ratio, ELR: eosinophil/lymphocyte ratio, BLR: basophil/lymphocyte ratio adolescents with ADHD. Our results showed that ELR and BLR were similar in both groups. This result is consistent with recent literature that investigated lymphocyte-related ratios (29). On the other hand, the PDW value was significantly higher in the ADHD group, compared to the control group. In other words, the increase in PDW in children and adolescents with ADHD is a new finding.

Other parameters interpreted as inflammatory markers include the changes in platelet features. Studies have shown that platelets play an important role in psychiatric disorders (30). In a single study investigating the level of PDW in psychiatric disorders, the PDW increase was found to be significant in patients with panic disorder as compared to the control group (26). The increase in PDW supports the immune system irregularity hypothesis in relation with ADHD in children and adolescents. On the other hand, the increase in PDW was found to be an independent predictor for myocardial infarction (31). The MPV is not only a useful predictor in the evaluation of systemic inflammation, but also a powerful predictor that shows the disease activity in a variety of inflammatory diseases $(24,32)$. In addition, MPV was reported to increase significantly in children with ADHD as compared to the control group (33). The increase in MPV which is considered as an inflammation marker was also demonstrated in this study. In other words, the MPV is an important risk factor in terms of cardiovascular disease (34). Yorbik et al. hypothesized that childhood ADHD may be a risk factor for coronary heart disease in adulthood (33). Similarly, in a study on the link between hyperactive behavior in childhood and cardiovascular risk in young adulthood, the childhood hyperactivity was associated with increased carotid artery intima media thickness (35). Both studies blame atherosclerotic changes among the underlying causes of the increased cardiovascular risk. Chronic inflammation causes the development of atherosclerosis through proinflammatory cytokines, which can pose a risk of cardiovascular disease in the future (36). Therefore, increased PDW and MPV values may be useful markers for early detection of possible cardiovascular complications that may occur as a result of chronic inflammation in later ages in children and adolescents with ADHD. 


\section{6-262}

It was also reported that the combined subtype diagnosis was more predominant in patients admitted to the clinic while the hyperactive/impulsive subtype was the least common (3). The present study is in line with the literature in terms of the distribution of ADHD group subtypes. A significant difference was found only in the MPV value, when hematological inflammatory markers were compared between ADHD subtypes. While there was no significant difference between the inattentive subtype and combined subtype, there was a statistically significant difference between hyperactivity/impulsivity and inattention. Unlike our findings, there was no significant relationship between the MPV and ADHD subtypes in Avcil's study (27). However, it is believed that a direct comparison could not be made between the subtypes due to the small number of samples in their study. Although the association between inflammation and ADHD has been reported, there are no studies investigating the differences in inflammation between all three subtypes. In a study that investigates the inflammatory cytokines in ADHD, no difference was reported between IL-6 and TNF-a between combined and inattentive subtypes (37). This result supports the conclusion of our study that there was no difference between inattentive and combined subtypes. On the other hand, a positive relationship was shown between the MPV and IL-6 levels (34). In the present study, the difference in MPV levels between inattentive and hyperactive/impulsive subtypes is hypothesized to be due to the changes in the levels of IL-6 or other inflammatory cytokines. Further studies are needed to clarify the underlying mechanisms that may be causing this. Moreover, additional studies need to be conducted to reveal the relationship between ADHD and inflammation in all three subtypes.

When girls and boys were analyzed separately in the study group, girls with ADHD had significantly higher WBC and neutrophil counts and NLR, as compared to boys with ADHD. NLR was higher in females at younger ages than in males (38). Besides, in their study of patients with major depressive disorder, Adhikari et al. hypothesized that females exhibited a greater inflammatory response than males, and that an increase in NLR was associated with poor prognosis in females (39). All these findings suggest that females may be more prone to inflammation. It has been stated that females exhibit a more proinflammatory condition compared to males, and are more likely to develop an autoimmune disease (40). Additionally, it has been noted that there is a positive relationship between ADHD and immune-related diseases in females while gender-specific immune-mediated neurodevelopmental processes may be involved in the ADHD etiology (41).

There is growing evidence of a relationship between inflammation and ADHD in studies on the causes of ADHD. Cytokines function in the regulation of the immune system, while high cytokine levels associated with chronic inflammation affect fetal development either directly through the effect on the neuronal development or through epigenetic mechanisms resulting in gene expression (42). In a study investigating the relationships between serum cytokines in children and adolescents with ADHD, and pregnancy and perinatal characteristics, significant correlations were demonstrated between increased IFN-g, shortened duration of pregnancy and low birth weight, decreased TNF-a and increased pregnancy-related problems, in addition to increased IL-6 and paternal smoking (43). In addition, the increase in cytokine release due to an inflammatory process may affect the maturation of the prefrontal cortex in individuals with ADHD (44). These studies show that the immune system activation may play a role in the etiology of neurodevelopmental disorders such as ADHD. However, because of the high costs and limited availability, inflammatory cytokine levels in patients with ADHD are unlikely to be examined routinely. In contrast, hematological inflammatory ratios have advantages in both low cost and availability as compared to other markers of inflammation, such as cytokines. Inflammatory ratios give information about innate and adaptive systems that are the components of the immune system, and reflect a reliable measurement of the inflammatory condition. Therefore, we believe that hematological parameters can be used when investigating the role of inflammation in the etiology of ADHD.

Although this current study shows that inflammatory processes play an important role in ADHD etiology, it still has some limitations. Our first and most important limitation is that the retrospective design of the study did not allow for a structured psychiatric interview to be carried out. Secondly, the possible correlations between symptom severity and duration of illness and inflammatory markers could not be assessed. Thirdly, other inflammatory markers such as inflammatory cytokines and acute-phase reactants were not evaluated. Fourthly, although the two groups were similar in age, gender, and BMI, one potential limitation was the inability to evaluate the uncontrollable factors such as the genetic characteristics, lifestyle and diet of the children and adolescents. On the other hand, the large sample size together with the facts that the subjects were taking no drugs and had no comorbidities are the major strengths of the study. As far as we know, it is also the first study that investigated the values considered as markers of inflammation, such as ELR, BLR, and PDW, in children and adolescents with ADHD.

\section{Conclusion}

As a result, this study shows that NLR, PLR, PDW, and MPV values, as well as WBC and neutrophil counts are significantly higher in children and adolescents with ADHD than in healthy controls, and that there is an association between low-grade inflammation and ADHD. Hematologic parameters may be useful markers for early detection of possible cardiovascular complications that may occur as a result of chronic inflammation at later age in children and adolescents with ADHD. The facts of higher WBC and neutrophil counts and higher NLR values in girls with ADHD, as compared to boys with ADHD, suggest that girls may be more prone to inflammation. In addition, prospective studies are needed to determine whether hematologic inflammatory marker levels would change after ADHD treatment. Also, we believe that these markers in ADHD should be investigated if they are correlated with cytokines and other markers of inflammation. 


\section{References}

1. American Psychiatric Association. Diagnostic and Statistical Manual of Mental Disorders, 5th Edition: DSM-5. American Psychiatric Publishing; 2013.

2. Polanczyk G, De Lima MS, Horta BL, Biederman J, Rohde LA. The Worldwide Prevalence of ADHD: A Systematic Review and Metaregression Analysis. Am J Psychiatry 2007; 164 (6): 942-948.

3. Willcutt EG. The Prevalence of DSM-IV Attention-Deficit/Hyperactivity Disorder: A Meta-Analytic Review. Neurotherapeutics 2012; 9 (3): 490-499.

4. Thapar A, Cooper M, Eyre O, Langley K. Practitioner review: What have we learnt about the causes of ADHD? J Child Psychol Psychiatry Allied Discip 2013; 54 (1): 3-16.

5. Strickland AD. Prevention of cerebral palsy, autism spectrum disorder, and attention deficit - Hyperactivity disorder. Med Hypotheses [Internet] 2014; 82 (5): 522-528. Available from: http://dx.doi.org/10.1016/j. mehy.2014.02.003

6. Frans EM. The Importance of Immune System Diseases in the Etiology of Attention-Deficit/Hyperactivity Disorder. Biol Psychiatry [Internet] 2017; 81 (5): e39-40. Available from: http://dx.doi.org/10.1016/j. biopsych.2016.12.016

7. Nielsen PR, Benros ME, Dalsgaard S. Associations Between Autoimmune Diseases and Attention-Deficit/Hyperactivity Disorder: A Nationwide Study. J Am Acad Child Adolesc Psychiatry [Internet] 2017; 56 (3): 234-240.e1. Available from: http://dx.doi.org/10.1016/j.jaac.2016.12.010

8. Mitchell RHB, Goldstein BI. Inflammation in children and adolescents with neuropsychiatric disorders: A systematic review. J Am Acad Child Adolesc Psychiatry [Internet] 2014; 53 (3): 274-296. Available from: http:// dx.doi.org/10.1016/j.jaac.2013.11.013

9. Oades RD, Myint AM, Dauvermann MR, Schimmelmann BG, Schwarz MJ. Attention-deficit hyperactivity disorder (ADHD) and glial integrity: An exploration of associations of cytokines and kynurenine metabolites with symptoms and attention. Behav Brain Funct 2010; 6:1-19.

10. Oades RD, Dauvermann MR, Schimmelmann BG, Schwarz MJ, Myint AM. Attention-deficit hyperactivity disorder (ADHD) and glial integrity: S100B, cytokines and kynurenine metabolism-effects of medication. Behav Brain Funct 2010; 6 (1): 29.

11. Felger JC, Miller AH. Cytokine Effects on the Basal Ganglia and Dopamine Function: the Subcortical Source of Inflammatory Malaise. Front Neuroendocr 2012; 33 (3): 315-327.

12. Mazza MG, Tringali AGM, Rossetti A, Botti RE, Clerici M. Crosssectional study of neutrophil-lymphocyte, platelet-lymphocyte and monocyte-lymphocyte ratios in mood disorders. Gen Hosp Psychiatry [Internet] 2019; 58 (February): 7-12. Available from: https://doi.org/10.1016/j.genhosppsych.2019.02.003

13. Zahorec R. Ratio of neutrophil to lymphocyte counts--rapid and simple parameter of systemic inflammation and stress in critically ill. Bratisl Lek Listy 2001; 102 (1): 5-14.

14. Erre GL, Paliogiannis P, Castagna F et al. Meta-analysis of neutrophil-to-lymphocyte and platelet-to-lymphocyte ratio in rheumatoid arthritis. Eur J Clin Invest 2019; 49 (1): 1-11.

15. Angkananard T, Anothaisintawee T, McEvoy M, Attia J, Thakkinstian A. Neutrophil Lymphocyte Ratio and Cardiovascular Disease Risk: A Systematic Review and Meta-Analysis. Biomed Res Int 2018; 2018.
16. Özdin S, Sarisoy G, Böke Ö. A comparison of the neutrophil-lymphocyte, platelet-lymphocyte and monocyte-lymphocyte ratios in schizophrenia and bipolar disorder patients - a retrospective file review. Nord J Psychiatry [Internet] 2017; 71 (7): 509-512. Available from: https://doi. org/10.1080/08039488.2017.1340517

17. Mazza MG, Lucchi S, Tringali AGM, Rossetti A, Botti ER, Clerici M. Neutrophil/lymphocyte ratio and platelet/lymphocyte ratio in mood disorders: A meta-analysis. Prog Neuro-Psychopharmacology Biol Psychiatry [Internet] 2018; 84: 229-236. Available from: https://doi.org/10.1016/j. pnpbp.2018.03.012

18. Kirlioglu SS, Balcioglu YH, Kalelioglu T, Erten E, Karamustafalioglu N. Comparison of the complete blood count-derived inflammatory markers in bipolar patients with manic and mixed episodes. Bratisl Lek Listy 2019; 120 (3): 195-199.

19. Özyurt G, Binici NC. Increased neutrophil-lymphocyte ratios in depressive adolescents is correlated with the severity of depression. Psychiatry Res 2018; 268: 426-431.

20. Özyurt G, Binici NC. The neutrophil-lymphocyte ratio and plateletlymphocyte ratio in adolescent obsessive-compulsive disorder: Does comorbid anxiety disorder affect inflammatory response? Psychiatry Res [Internet] 2019; 272 (February 2018): 311-315. Available from: https:// doi.org/10.1016/j.psychres.2018.12.131

21. Dietrich-Muszalska A, Wachowicz B. Platelet haemostatic function in psychiatric disorders: Effects of antidepressants and antipsychotic drugs. World J Biol Psychiatry 2017; 18 (8): 564-574.

22. Balta S, Demirkol S, Kucuk U. The platelet lymphocyte ratio may be useful inflammatory indicator in clinical practice. Hemodial Int 2013; 4 (17): 668-669.

23. Inanli I, Aydin M, Çaliskan AM, Eren I. Neutrophil/lymphocyte ratio, monocyte/lymphocyte ratio, and mean platelet volume as systemic inflammatory markers in different states of bipolar disorder. Nord J Psychiatry [Internet] 2019; 73 (6): 372-9. Available from: https://doi.org/10. 1080/08039488.2019.1640789

24. Balta S, Demirkol S, Cakar M, Celik T. Mean platelet volume as a surrogate marker of low-grade inflammation in osteoarthritis. Platelets 2014; 25 (8): 643-644.

25. Orak M, Karakoç Y, Ustundag M, Yildirim Y, Celen M, Güloglu C. An investigation of the effects of the mean platelet volume, platelet distribution width, platelet/lymphocyte ratio, and platelet counts on mortality in patents with sepsis who applied to the emergency department. Niger J Clin Pract 2018; 21 (5): 667-671.

26. Ransing RS, Patil B, Grigo O. Mean Platelet Volume and Platelet Distribution Width Level in Patients with Panic Disorder. J Neurosci Rural Pract 2017; 8 (2): 174.

27. Avcil S. Evaluation of the neutrophil/lymphocyte ratio, platelet/lymphocyte ratio, and mean platelet volume as inflammatory markers in children with attention-deficit hyperactivity disorder. Psychiatry Clin Neurosci 2018; 72 (7): 522-530.

28. Cevher Binici N, Kutlu A. Is ADHD an inflammation-related disorder? Anadolu Psikiyatr Derg 2019; 20 (3): 313-320.

29. Kara MZ, Orum MH, Egilmez OB. Relationship between immune cells and violent/nonviolent suicide attempts and controls: What about the lymphocyte-related ratios and neutrophil-related parameters? Kaohsiung J Med Sci 2019; 35 (5): 315-316. 


\section{$256-262$}

30. Wysokiński A, Szczepocka E. Platelet parameters (PLT, MPV, PLCR) in patients with schizophrenia, unipolar depression and bipolar disorder. Psychiatry Res 2016; 237: 238-245.

31. Cetin M, Bakirci EM, Baysal E et al. Increased platelet distribution width is associated with ST-segment elevation myocardial infarction and thrombolysis failure. Angiology 2014; 65 (8): 737-743.

32. Yuri Gasparyan A, Ayvazyan LP, Mikhailidis DD, Kitas G. Mean Platelet Volume: A Link Between Thrombosis and Inflammation? Curr Pharm Des 2011; 17 (1): 47-58.

33. Yorbik O, Mutlu C, Tanju IA, Celik D, Ozcan O. Mean platelet volume in children with attention deficit hyperactivity disorder. Med Hypotheses [Internet] 2014; 82 (3): 341-345. Available from: http://dx.doi. org/10.1016/j.mehy.2014.01.001

34. Chu SG, Becker RC, Berger PB et al. Mean platelet volume as a predictor of cardiovascular risk: A systematic review and meta-analysis. J Thromb Haemost 2010; 8 (1): 148-156.

35. Keltikangas-Järvinen L, Pulkki-Råback L, Puttonen S, Viikari J, Raitakari OT. Childhood hyperactivity as a predictor of carotid artery intima media thickness over a period of 21 years: The Cardiovascular Risk in Young Finns study. Psychosom Med 2006; 68 (4): 509-516.

36. Aloi M, Tromba L, Rizzo V et al. Aortic Intima-Media Thickness as an Early Marker of Atherosclerosis in Children with Inflammatory Bowel Disease. J Pediatr Gastroenterol Nutr 2015; 61 (1): 41-46.

37. Corominas-Roso M, Armario A, Palomar G et al. IL- 6 and TNF- $\alpha$ in unmedicated adults with ADHD: Relationship to cortisol awakening response. Psychoneuroendocrinology [Internet] 2017; 79: 67-673. Available from: http://dx.doi.org/10.1016/j.psyneuen.2017.02.017
38. Howard R, Scheiner A, Kanetsky PA, Egan KM. Sociodemographic and lifestyle factors associated with the neutrophil-to-lymphocyte ratio. Ann Epidemiol [Internet] 2019; 38: 11-21.e6. Available from: https://doi. org/10.1016/j.annepidem.2019.07.015

39. Adhikari A, Dikshit R, Karia S, Sonavane S, Shah N, De Sousa A. Neutrophil-lymphocyte ratio and c-reactive protein level in patients with major depressive disorder before and after pharmacotherapy. East Asian Arch Psychiatry 2018; 28 (2): 53-58.

40. Quintero OL, Amador-Patarroyo MJ, Montoya-Ortiz G, RojasVillarraga A, Anaya JM. Autoimmune disease and gender: Plausible mechanisms for the female predominance of autoimmunity. J Autoimmun [Internet] 2012; 38 (2-3): J109-119. Available from: http://dx.doi. org/10.1016/j.jaut.2011.10.003

41. Hegvik TA, Instanes JT, Haavik J, Klungsøyr K, Engeland A. Associations between attention-deficit/hyperactivity disorder and autoimmune diseases are modified by sex: a population-based cross-sectional study. Eur Child Adolesc Psychiatry 2018; 27 (5): 663-675.

42. Jasoni CL, Sanders TR, Kim DW. Do all roads lead to Rome? The role of neuro-immune interactions before birth in the programming of offspring obesity. Front Neurosci 2015; 9 (FEB): 1-13.

43. Oades RD. An exploration of the associations of pregnancy and perinatal features with cytokines and tryptophan/kynurenine metabolism in children with attention-deficit hyperactivity disorder (ADHD). ADHD Atten Deficit Hyperact Disord 2011; 3 (4): 301-318.

44. Buske-Kirschbaum A, Schmitt J, Plessow F, Romanos M, Weidinger S, Roessner V. Psychoendocrine and psychoneuroimmunological mechanisms in the comorbidity of atopic eczema and attention deficit/hyperactivity disorder. Psychoneuroendocrinology [Internet] 2013; 38 (1): 12-23. Available from: http://dx.doi.org/10.1016/j.psyneuen.2012.09.017

Received September 23, 2020. Accepted October 2, 2020. 